\title{
Aircraft Longitudinal Auto-landing Guidance Law Using Time Delay Control Scheme
}

\author{
By Hyoung Sik CHOI, ${ }^{1)}$ Sangjong LEE,${ }^{1)}$ Jangho LEE, ${ }^{1)}$ Eung Tai $\mathrm{KIM}^{1)}$ and Hyunchul SHIM ${ }^{2)}$ \\ ${ }^{1)}$ Korea Aerospace Research Institute, Daejeon, Republic of Korea \\ ${ }^{2)}$ Department of Aerospace Engineering, Korea Advanced Institute of Science and Technology, \\ Daejeon, Republic of Korea
}

(Received September 22nd, 2009)

\begin{abstract}
The objective of this paper is applying the Time Delay Control scheme for the aircraft auto-landing guidance problem. A stability and control augmentation system and longitudinal auto-landing guidance law, using Time Delay Control, is proposed and evaluated through a simulation with model uncertainties and wind disturbances. The proposed Time Delay Control guidance law shows good performance and is robust to model uncertainties and disturbances.
\end{abstract}

Key Words: Aircraft, Guidance and Control, Auto-landing, Time Delay Control, Turbulence

\section{Nomenclature}

$V_{T}:$ airspeed

$\alpha, \beta$ : angle of attack and sideslip

$C_{\mathrm{L}, \mathrm{D}, \mathrm{M}}$ : lift, drag, pitching moment coefficient

$h$ : altitude

$\phi, \theta, \psi$ : roll, pitch, and yaw angle

$p, q, r$ : roll, pitch, and yaw rate

$\bar{L}, \bar{T}:$ total lift and thrust force

$\bar{D}, \bar{Y}:$ total drag and side force

$\bar{l}$ : total rolling moment

$\bar{M}$ : total pitching moment

$\bar{N}$ : total yawing moment

$M_{\delta_{\mathrm{e}}}$ : elevator control moment

$l_{\delta_{\mathrm{a}}}:$ aileron control moment

$I_{x, y, z}$ : moment of inertia in roll, pitch, and yaw axis

$I_{x z}:$ moment of inertia in coupled axis

$\delta_{\mathrm{a}}, \delta_{\mathrm{e}}$ : aileron and elevator deflection angle

$\gamma$ : flight path angle

$m, m g$ : aircraft mass and total weight

$n$ : aircraft forward ground track

$L:$ small time step

$t$ : time

$\boldsymbol{e}$ : error vector

$\boldsymbol{u}$ : control input vector

$x$ : states vector

Subscripts

0 : initial

d: desired

glide: desired glide slope trajectory

flare: desired flare trajectory

\section{Introduction}

This paper proposes an implementation of the Time Delay Control (TDC) scheme for the aircraft automatic

(C) 2010 The Japan Society for Aeronautical and Space Sciences landing guidance problem. The TDC scheme has been widely used in robotics and is a successful control scheme for many nonlinear systems since it was first proposed. ${ }^{1)}$ In particular, the TDC scheme is known as a robust nonlinear control scheme for model uncertainties and displays positive performance for the trajectory tracking problem. ${ }^{2,3)}$

Several previous studies on longitudinal auto-landing control are well summarized by Ju and Tsai ${ }^{4)}$ who implemented an adaptive backstepping control scheme. Backstepping control is based on Lyapunov Stability and requires strict feedback equation forms of the system's dynamic model, which can be quite complicated for developing control law. One nonlinear control approach for auto-landing, named dynamic model inversion, is described by $\mathrm{Ha}$ et al. ${ }^{5)}$ who applied the singular perturbation technique. A dynamic model inversion scheme requires full model properties, rendering itself vulnerable to model uncertainty. Consequently, such a model inversion scheme generally requires an additional controller, such as a neural network, to compensate for model uncertainties.

However, the TDC control approach has several advantages compared to previous nonlinear approaches. As mentioned before, TDC is robust for model uncertainties and disturbances. Also, the TDC control law can be determined easily by adopting the phase variable equation forms if the target system can be considered as rigid and controllable. In addition, its control methodology is similar to a human being, making TDC easy to understand, develop, implement, and modify. The ease of use can be attributed to the use of desired dynamics with estimated control effectiveness, one step previous states, control history, and not requiring an accurate system model.

\section{Nonlinear Dynamics and TDC}

\subsection{Aircraft dynamics}

The general aircraft 6-DOF nonlinear flight dynamic 
equations of motion are shown below. ${ }^{6,7)}$ The dynamics have cross-coupling terms, and aerodynamic coefficients are established by nonlinear look-up tables.

$$
\begin{aligned}
\dot{V}_{T}= & {[\bar{T} \cos \alpha \cos \beta-\bar{D} \cos \beta+\bar{Y} \sin \beta} \\
& +m g(-\cos \alpha \cos \beta \sin \theta+\sin \beta \sin \phi \cos \theta \\
& +\sin \alpha \cos \beta \cos \phi \cos \theta)] / m \\
\dot{\alpha}= & {\left[-\bar{T} \sin \alpha-\bar{L}+m V_{T}(-p \cos \alpha \sin \beta+q \cos \beta\right.} \\
& -r \sin \alpha \sin \beta)+m g(\sin \alpha \sin \theta \\
& +\cos \alpha \cos \phi \cos \theta)] / m V_{T} \cos \beta \\
\dot{\beta}= & {[-\bar{T} \cos \alpha \sin \beta+\bar{D} \sin \beta+\bar{Y} \cos \beta} \\
& -m V_{T}(-p \sin \alpha+r \cos \alpha)+m g(\cos \alpha \sin \beta \sin \theta \\
& +\cos \beta \sin \phi \cos \theta-\sin \alpha \sin \beta \cos \phi \cos \theta)] / m V_{T} \\
\dot{h}= & V_{T}(\cos \alpha \cos \beta \sin \theta-\sin \beta \sin \phi \cos \theta \\
& -\sin \alpha \cos \beta \cos \phi \cos \theta) \\
\dot{\theta}= & q \cos \phi-r \sin \phi \\
\dot{\phi}= & p+q \sin \phi \tan \theta+r \cos \phi \tan \theta \\
\dot{\psi}= & q \sin \phi / \cos \theta+r \cos \phi / \cos \theta \\
\dot{p}= & C_{3} \bar{l}+C_{4} \bar{N}+\left(C_{1} r+C_{2} p\right) q \\
\dot{q}= & C_{7} \bar{M}+C_{5} p r-C_{6}\left(p^{2}-r^{2}\right) \\
\dot{r}= & C_{9} \bar{N}+C_{4} \bar{l}+\left(C_{8} p-C_{2} r\right) q
\end{aligned}
$$

where moment of inertia terms are:

$$
\begin{aligned}
& C_{0}=I_{x} I_{z}-I_{x z}{ }^{2}, \quad C_{1}=\left[\left(I_{y}-I_{z}\right) I_{z}-I_{x z}{ }^{2}\right] / C_{0}, \\
& C_{2}=\left[\left(I_{x}-I_{y}+I_{z}\right) I_{x z}\right] / C_{0}, \quad C_{3}=I_{z} / C_{0}, \\
& C_{4}=I_{x z} / C_{0}, \quad C_{5}=\left(I_{z}-I_{x}\right) / I_{y}, \quad C_{6}=I_{x z} / I_{y}, \\
& C_{7}=1 / I_{y}, \quad C_{8}=\left[I_{x}\left(I_{x}-I_{y}\right)+I_{x z}{ }^{2}\right] / C_{0}, \\
& C_{9}=I_{x} / C_{0}
\end{aligned}
$$

\subsection{Time delay control (TDC)}

The TDC control scheme has been well described elsewhere. ${ }^{8)}$ For convenience, it is reviewed below.

Consider a general nonlinear dynamic equation as Eq. (11),

$$
\dot{\boldsymbol{x}}=\boldsymbol{f}(\boldsymbol{x}, t)+\boldsymbol{B}(\boldsymbol{x}, t) \boldsymbol{u}+\boldsymbol{d}(t)
$$

where $\boldsymbol{d}(t)$ denotes disturbance. To develop the control law, the error dynamics can be defined as Eq. (12),

$$
\dot{\boldsymbol{e}}_{\mathrm{d}}=\boldsymbol{A}_{\mathrm{d}} \boldsymbol{e}_{\mathrm{d}}
$$

where $\boldsymbol{e}_{\mathrm{d}}=\boldsymbol{x}_{\mathrm{d}}-\boldsymbol{x}$.

Equation (11) can be rewritten as Eq. (13).

$$
\dot{\boldsymbol{x}}=\hat{\boldsymbol{f}}(\boldsymbol{x}, t)+\hat{\boldsymbol{B}} \boldsymbol{u}
$$

where $\hat{\boldsymbol{B}}$ denotes estimated control effectiveness which is a constant matrix (or value) representing the known range of $\boldsymbol{B}(\boldsymbol{x}, t)$. Thus, $\hat{\boldsymbol{f}}(\boldsymbol{x}, t)$ can be rearranged as:

$$
\hat{\boldsymbol{f}}(\boldsymbol{x}, t)=\boldsymbol{f}(\boldsymbol{x}, t)+[\boldsymbol{B}(\boldsymbol{x}, t)-\hat{\boldsymbol{B}}] \boldsymbol{u}+\boldsymbol{d}(t)
$$

Eq. (14) contains all the nonlinear uncertainties and disturbance terms. With Eq. (11) and Eq. (12), the error dynamics can be expressed as Eq. (15).

$$
\dot{\boldsymbol{e}}_{\mathrm{d}}=\dot{\boldsymbol{x}}_{\mathrm{d}}-\dot{\boldsymbol{x}}=\dot{\boldsymbol{x}}_{\mathrm{d}}-\hat{\boldsymbol{f}}(\boldsymbol{x}, t)-\hat{\boldsymbol{B}} \boldsymbol{u}
$$

Comparing with Eq. (12), the computed control input is given as Eq. (16).

$$
\boldsymbol{u}=\hat{\boldsymbol{B}}^{-1}\left[-\hat{\boldsymbol{f}}(\boldsymbol{x}, t)+\dot{\boldsymbol{x}}_{\mathrm{d}}-\boldsymbol{A}_{\mathrm{d}} \boldsymbol{e}_{\mathrm{d}}\right]
$$

If desired dynamics is defined as $\dot{\boldsymbol{x}}_{\mathrm{d}}=\boldsymbol{A}_{\mathrm{d}} \boldsymbol{x}_{\mathrm{d}}+\boldsymbol{B}_{\mathrm{d}} \boldsymbol{r}$, Eq. (16) can be expressed as Eq. (17).

$$
\boldsymbol{u}=\hat{\boldsymbol{B}}^{-1}\left[-\hat{\boldsymbol{f}}(\boldsymbol{x}, t)+\boldsymbol{A}_{\mathrm{d}} \boldsymbol{x}+\boldsymbol{B}_{\mathrm{d}} \boldsymbol{r}\right]
$$

Using time delay estimation as Eq. (18) on the assumption that the time step, $L$, is very small,

$$
\hat{\boldsymbol{f}}(\boldsymbol{x}, t) \cong \hat{\boldsymbol{f}}(\boldsymbol{x}, t-L)
$$

Eq. (13) can be rewritten as Eq. (19).

$$
\hat{\boldsymbol{f}}(\boldsymbol{x}, t) \cong \dot{\boldsymbol{x}}(t-L)-\hat{\boldsymbol{B}} \boldsymbol{u}(t-L)
$$

Substituting Eq. (19) into Eq. (17), the uncertainty terms can be canceled. Finally, the computed control input is obtained as Eq. (20).

$$
\boldsymbol{u}=\boldsymbol{u}(t-L)+\hat{\boldsymbol{B}}^{-1}\left[-\dot{\boldsymbol{x}}(t-L)+\boldsymbol{A}_{\mathrm{d}} \boldsymbol{x}+\boldsymbol{B}_{\mathrm{d}} \boldsymbol{r}\right]
$$

Equation (20) is a typical TDC control law. It does not require an exact dynamic model and its properties because there are no internal plant dynamic equations. It uses previous step input, states, and estimated control effectiveness with pre-shaped input for desired output.

\section{TDC Stability/Control Augmentation System}

This section describes TDC for Stability/Control Augmentation System (SCAS) design. The 6-DOF equations of motion can be composed into four subsystems, such as airspeed, pitch, roll, and yaw channel. For simplicity, the airspeed and yaw channel can be controlled by classical PI controllers. Therefore, it can be assumed that airspeed is constant and sideslip angle and yaw rate are zero.

\subsection{Pitch stability/control augmentation system}

From the nonlinear equations in Eq. (5) and Eq. (9), the pure pitch dynamics in the longitudinal motion can be:

$$
\begin{aligned}
& \dot{\theta}=q \\
& \dot{q}=\frac{1}{I_{y}} \bar{M}\left(q, \delta_{\mathrm{e}}, \alpha, \dot{\alpha}, \ldots\right)
\end{aligned}
$$

where $\bar{M}$ is the total pitching moment consisting of combinational terms of various states and nonlinear tables. Therefore, it shows strong nonlinear characteristics. Equations (21) and (22) can be rearranged as one formula in Eq. (23) with disturbance $d(t)$.

$$
I_{y} \ddot{\theta}=M_{\mathrm{q}} q+M_{\delta_{\mathrm{e}}} \delta_{\mathrm{e}}+M_{\alpha} \alpha+M_{\dot{\alpha}} \dot{\alpha}+d(t)+\ldots
$$

If the angle of attack and other terms can be considered as disturbances, Eq. (23) can be expressed as Eq. (24). 


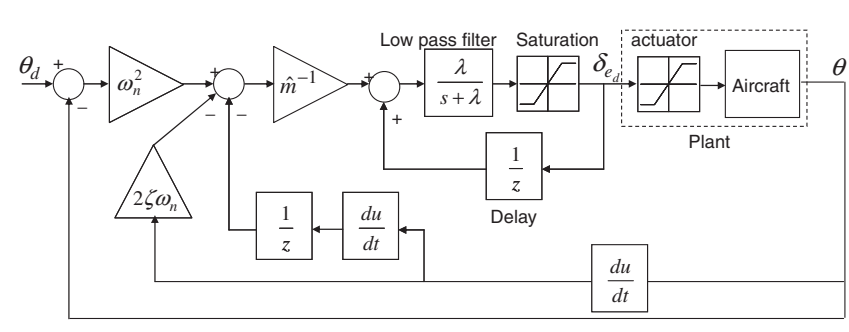

Fig. 1. Pitch channel SCAS.

$$
\begin{aligned}
\left\{\begin{array}{c}
\dot{\theta} \\
\ddot{\theta}
\end{array}\right\}= & {\left[\begin{array}{cc}
0 & 1 \\
0 & M_{\mathrm{q}}{ }^{\prime}
\end{array}\right]\left\{\begin{array}{l}
\theta \\
\dot{\theta}
\end{array}\right\}+\left[\begin{array}{c}
0 \\
M_{\delta_{\mathrm{e}}}{ }^{\prime}
\end{array}\right] \delta_{\mathrm{e}} } \\
& +\left[\begin{array}{c}
0 \\
d(\alpha, \dot{\alpha}, t, \ldots)
\end{array}\right]
\end{aligned}
$$

where $M_{\mathrm{O}}{ }^{\prime}=M_{\mathrm{O}} / I_{y}$.

From Eq. (23), the control input is obtained as:

$$
\delta_{\mathrm{e}}=\frac{1}{\hat{M}_{\delta_{\mathrm{e}}}{ }^{\prime}} \ddot{\theta}-\hat{f}
$$

where $\hat{f}=M_{\mathrm{q}}{ }^{\prime} \dot{\theta}+M_{\delta_{\mathrm{e}}}{ }^{\prime} \delta_{\mathrm{e}}-\hat{M}_{\delta_{\mathrm{e}}}{ }^{\prime} \delta_{\mathrm{e}}+d(\alpha, \dot{\alpha}, t, \ldots), \hat{M}_{\delta_{\mathrm{e}}}{ }^{\prime}=$ $\hat{M}_{\delta_{\mathrm{e}}} / \hat{I}_{y}$.

Defining the desired pitch angle as $\theta_{\mathrm{d}}$, the desired reference model can be determined as Eq. (26).

$$
\left\{\begin{array}{c}
\dot{\theta} \\
\ddot{\theta}
\end{array}\right\}=\left[\begin{array}{cc}
0 & 1 \\
-\omega_{\mathrm{nd}}^{2} & -2 \zeta_{\mathrm{d}} \omega_{\mathrm{nd}}
\end{array}\right]\left\{\begin{array}{c}
\theta \\
\dot{\theta}
\end{array}\right\}+\left[\begin{array}{c}
0 \\
\omega_{\mathrm{nd}}{ }^{2}
\end{array}\right] \theta_{\mathrm{d}}
$$

Equation (26) is equivalent to $\ddot{\theta}+2 \zeta_{\mathrm{d}} \omega_{\mathrm{nd}} \dot{\theta}+\omega_{\mathrm{nd}}^{2} \theta=$ $\omega_{\text {nd }}^{2} \theta_{\mathrm{d}}$, where $\zeta_{\mathrm{d}}$ is the damping ratio and $\omega_{\text {nd }}$ is the natural frequency of the desired dynamics. Finally, the TDC control law for the pitch channel can be obtained as Eq. (27).

$$
\begin{aligned}
\delta_{\mathrm{e}}= & \delta_{\mathrm{e}}(t-L)+\hat{m}^{-1}\left[-\ddot{\theta}(t-L)+\omega_{\mathrm{nd}}^{2}\left(\theta_{\mathrm{d}}-\theta\right)\right. \\
& \left.-2 \zeta_{\mathrm{d}} \omega_{\mathrm{nd}} \dot{\theta}\right]
\end{aligned}
$$

where $\hat{m}=\hat{M}_{\delta_{\mathrm{e}}{ }^{\prime}}=\frac{\hat{M}_{\delta_{\mathrm{e}}}}{\hat{I}_{y}}$

In Eq. (27), the only required model property is estimated control effectiveness $\hat{m}$. Other terms are previous control input $\delta_{\mathrm{e}}(t-L)$, second derivative of previous state $\ddot{\theta}(t-L)$, current feedback state $\theta$, and desired dynamics $\theta_{\mathrm{d}}, \zeta_{\mathrm{d}}, \omega_{\mathrm{nd}}$, which are all known.

Figure 1 shows the block diagram of the pitch SCAS of TDC. Both the damping ratio and natural frequency of desired dynamics should be determined during tuning. Here, the desired damping ratio is 0.9 and the natural frequency is $3 \mathrm{rad} / \mathrm{s}$. The low-pass filter and saturation blocks are inserted as shown in Fig. 1. The saturation filter prevents control actuator windup. ${ }^{9)}$ Additionally, a low-pass filter is required to eliminate the high-frequency term induced by the double derivative of $\theta \cdot{ }^{10)}$ Here, the cutoff frequency $\lambda$ of the low-pass filter is $4 \mathrm{rad} / \mathrm{s}$.

\subsection{Roll stability/control augmentation system}

The roll SCAS channel of TDC can be also designed in the same manner as described above. From Eqs. (6) and (8), the pure roll channel dynamics can be defined as Eq. (28).

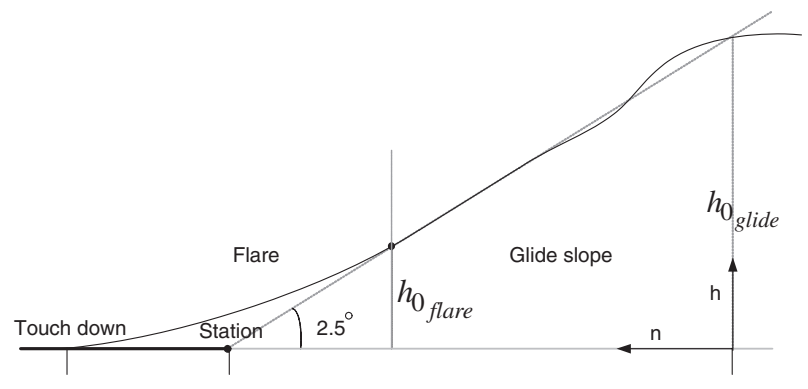

Fig. 2. Longitudinal landing flight path.

$$
\dot{\phi}=p, \quad \dot{p}=\frac{1}{I_{x}} \bar{l}\left(v, p, r, \delta_{\mathrm{a}}, \ldots\right)
$$

The TDC control law for the roll channel can be obtained as Eq. (29).

$$
\begin{aligned}
\delta_{\mathrm{a}}= & \delta_{\mathrm{a}}(t-L)+\hat{l}^{-1}\left[-\ddot{\phi}(t-L)+\omega_{\mathrm{n}}^{2}\left(\phi_{\mathrm{d}}-\phi\right)\right. \\
& \left.-2 \zeta \omega_{\mathrm{n}} \dot{\phi}\right]
\end{aligned}
$$

where $\hat{l}=\frac{\hat{l}_{\delta_{\mathrm{a}}}}{\hat{I}_{x}}$.

\section{Reference Trajectory Generation for Auto-landing}

To apply the TDC control to the auto-landing tracking problem, we must generate a desired trajectory. For a longitudinal landing flight path, two reference trajectories are necessary in the approach phase: the glide slope, and the flare as presented in Fig. 2. The flight path angle is defined as Eq. (30).

$$
\gamma=\theta-\alpha
$$

If the desired flight path angle $\gamma$ is determined, the desired pitch angle $\theta$ can be determined with the angle of attack $\alpha$.

Here, the angle of attack $\alpha$ is considered as a disturbance. If $\alpha$ changes, the desired pitch angle $\theta_{\mathrm{d}}$ should be adjusted automatically with the constrained flight path angle $\gamma_{\mathrm{d}}$. Therefore, the desired pitch angle can be defined as Eq. (31).

$$
\theta_{\mathrm{d}}=\gamma_{\mathrm{d}}+\alpha
$$

Calculating the desired pitch angle requires the desired flight path angle and the detail procedures are explained below.

\subsection{Glide slope guidance}

The glide slope trajectory is a straight line with a given flight path angle. In general, the glide slope angle is $2.5^{\circ}-$ $3.0^{\circ}$. In this paper, the glide slope angle is $2.5^{\circ}$, so the desired flight path angle is $-2.5^{\circ}$. Whether the touch-down point is not determined or simple simulation evaluation is pursued, Eq. (31) can provide the desired pitch angle.

For practical purposes, it is necessary to guide the aircraft to a specific station point for glide slope tracking as shown in Fig. 2. If there is a small tracking error in the flight path angle due to disturbance, the resultant touch-down point deviates in a large error boundary. Equation (31) is unsuit- 


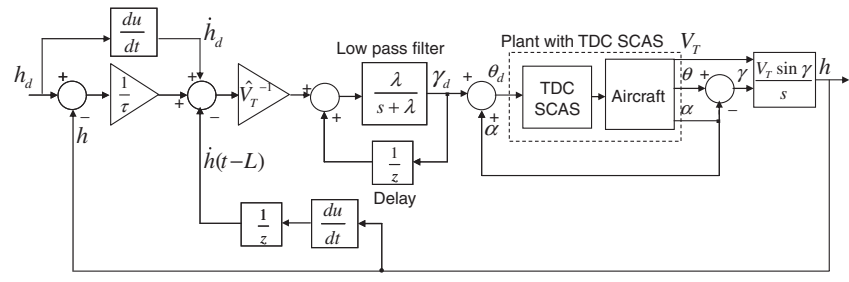

Fig. 3. Longitudinal glide slope guidance law.

able for this error. Therefore, a guidance law to the station point can be achieved by using altitude and ground position. The rate of altitude change can be defined as Eq. (32).

$$
\dot{h}=V_{T} \sin \gamma
$$

Applying the TDC concept, Eq. (32) can be reformulated as Eq. (33).

$$
\dot{h}=\hat{f}+\hat{V}_{T} \gamma
$$

where $\hat{f}=V_{T} \sin \gamma-\hat{V}_{T} \gamma+d(t)$.

If the error dynamic is defined as $\dot{e}=-\frac{1}{\tau} e$, then

$$
\dot{h_{\mathrm{d}}}-\dot{h}=\dot{h}_{\mathrm{d}}-\hat{V}_{T} \gamma-\hat{f}=-\frac{1}{\tau}\left(h_{\mathrm{d}}-h\right)
$$

Using time delay estimation, the TDC glide slope guidance control is obtained as Eq. (35).

$$
\gamma_{\text {glide }}=\gamma_{\text {glide }}(t-L)+\hat{V}_{T}^{-1}\left[\dot{h}_{\mathrm{d}}-\dot{h}(t-L)+\frac{1}{\tau}\left(h_{\mathrm{d}}-h\right)\right]
$$

where $h_{\mathrm{d}_{\text {glide }}}=-\tan \left(2.5^{\circ}\right) \cdot n+h_{0_{\text {glide }}}$.

Figure 3 shows the block diagram of the glide slope guidance law. If the altitude, ground position, and station point are known at the initial landing approach phase, the desired flight path angle can be determined easily. Here, the time constant $\tau$ for the error dynamic is $5 \mathrm{rad} / \mathrm{s}$ and the cutoff frequency $\lambda$ is $1 / 3 \mathrm{rad} / \mathrm{s}$.

\subsection{Flare guidance}

The desired flare altitude can be expressed as the exponential function ${ }^{7}$

$$
h_{\mathrm{d}_{\mathrm{flare}}}=h_{0_{\mathrm{flare}}} e^{-t / \tau}
$$

By applying $\dot{h}=V_{T} \sin \gamma \cong V_{T} \gamma$, Eq. (36) is solved as Eq. (37).

$$
\dot{h}=V_{T} \gamma=-h / \tau
$$

Finally, the desired flare flight path angle is determined as Eq. (38).

$$
\gamma_{\mathrm{d}_{\text {flare }}}=-h / V_{T} \tau
$$

The criteria for switching from glide slope phase to flare phase is altitude. Here, the altitude criteria for mode switching is $30 \mathrm{ft}$ and the time constant $\tau$ is $4.4 \mathrm{rad} / \mathrm{s}^{7}$ ) The resultant desired flight path angle of Eq. (38) was used as the input value directly in Eq. (31) instead of Eq. (35) in the flare phase.

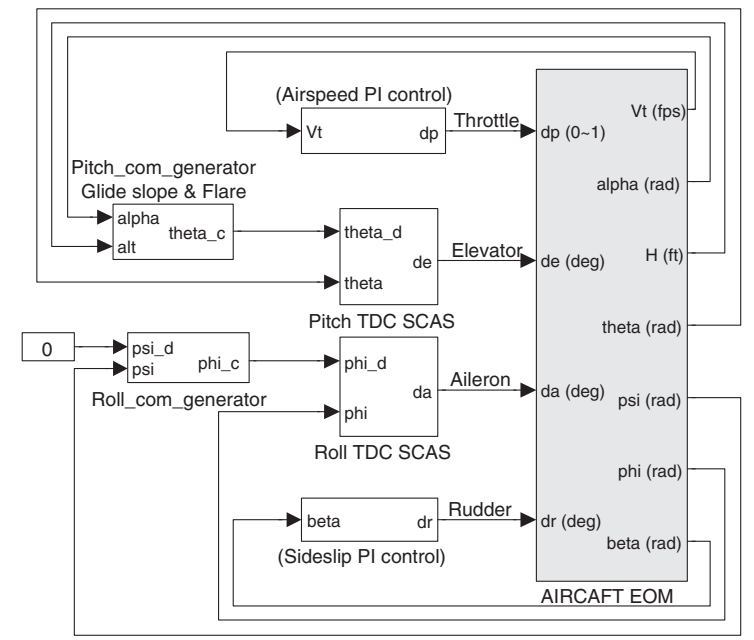

Fig. 4. Nonlinear aircraft dynamic model with TDC.

\section{Simulation Results}

An aircraft simulation model was developed using the MATLAB/Simulink environment. The full nonlinear 6DOF aircraft dynamics including actuator dynamics block, AIRCRAFT EOM, is on the right side of Fig. 4. The actuator bandwidth is $20 \mathrm{rad} / \mathrm{s}$. The simulation time step is 0.01 so that $L$ of Eq. (18) is 0.01 .

As described above, flight airspeed and sideslip angle are controlled by the PI controller shown in Fig. 4. Here, the roll channel SCAS is used only for holding roll angle to zero. Figure 5 shows the longitudinal aerodynamic coefficient tables. The aircraft has two elevator control surfaces divided into left ( $\left.\mathrm{de}_{-} \mathrm{L}\right)$ and right $\left(\mathrm{de} \_\mathrm{R}\right)$ elevator. The total aerodynamic coefficient can be calculated by summing the each aerodynamic force and moment due to flap, and elevator deflections based on the clean configuration.

The performance of the proposed SCAS controller for TDC was evaluated using model uncertainty insertion, and the desired tracking performance of the landing guidance controller was demonstrated with wind turbulence.

\subsection{TDC SCAS with/without model uncertainties}

To evaluate the robustness to model uncertainties, the SCAS controller for TDC was simulated. The reference input for the desired pitch angle is a sine wave and model uncertainties were applied as follows:

(1) Uncertainty of CG (Center of Gravity) position

(2) Aerodynamic change due to flap deflection and stuck left elevator control surface

(3) Uncertainty of moment of inertia

Figure 6 shows the response for the nominal case when there are no model uncertainties. Nominal values are as following: $\mathrm{CG}$ at 25\% MAC (Mean Aerodynamic Chord), flap deflection angle of $0^{\circ}$, and nominal moment of inertia of $25,085 \mathrm{slug} / \mathrm{ft}^{2}$ respectively. As shown, the SCAS controller provides good performance tracking the desired pitch angle $\theta_{\mathrm{d}}$.

Figure 7 shows the simulation results for the SCAS controller for TDC with model uncertainties. Compared to the 


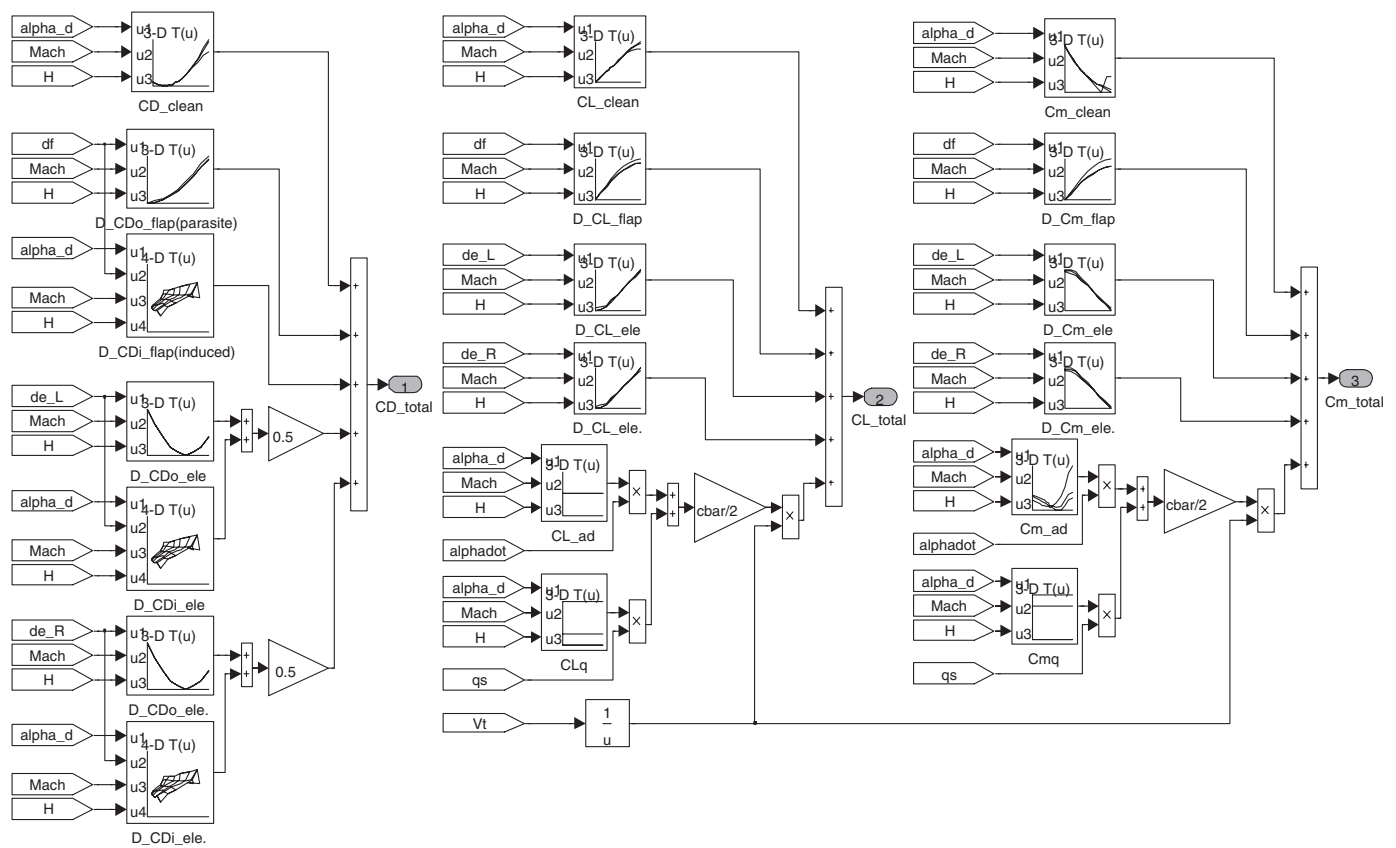

Fig. 5. Aerodynamic coefficients of aircraft.
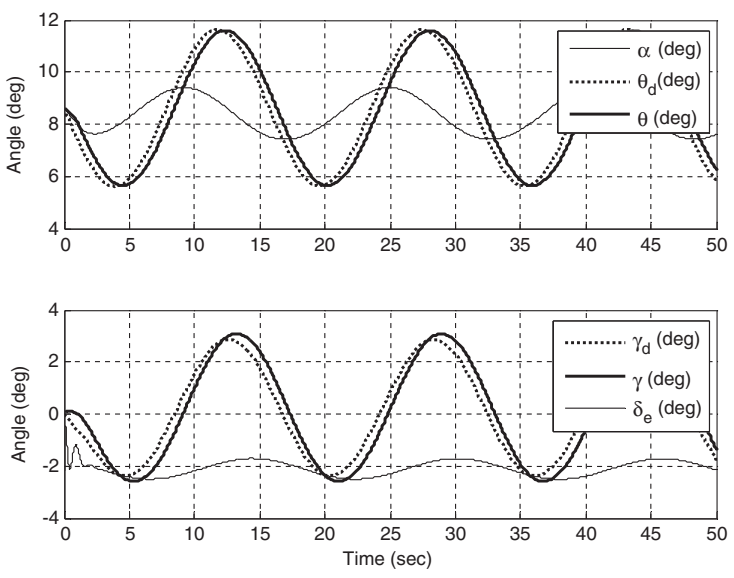

Fig. 6. SCAS of TDC simulation results for nominal case (without model uncertainties).

nominal case, the CG deviates to $35 \%$ MAC (nominal is $25 \% \mathrm{MAC}$ ) and $30^{\circ}$ flap deflection angle is applied. In addition, the moment of inertia has $10 \%$ error compared to the nominal value (nominal value $\times 0.9$ ) and the left elevator control surface is stuck at the initial trim position, affecting control effectiveness $\hat{m}$ in Eq. (27).

Flap deflection changes drag, lift, and pitching moment. The variation in aerodynamic coefficient due to flap deflection is:

$$
\begin{aligned}
& \text { - } \Delta C_{\mathrm{D}_{\text {Flap }}} / C_{\mathrm{D}_{\text {Total }}}=0.3551 \\
& \text { - } \Delta C_{\mathrm{L}_{\text {Fap }}} / C_{\mathrm{L}_{\text {Total }}}=0.2705 \\
& \text { - } \Delta C_{\mathrm{M}_{\text {Flap }}} / C_{\mathrm{M}_{\text {Total }}}=-0.2988
\end{aligned}
$$

Figure 7 shows the performance of the desired pitch angle tracking with model uncertainties. These results show that the proposed SCAS controller of TDC works well and it is robust to the unknown model uncertainties.
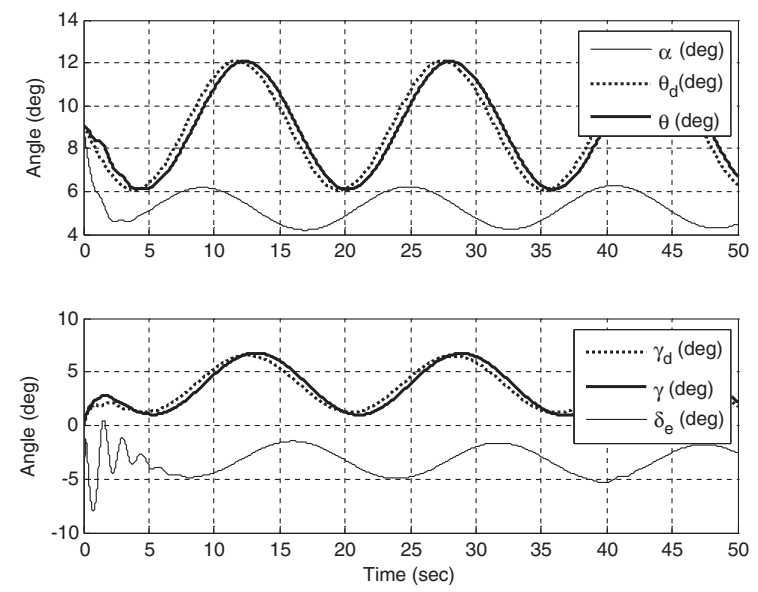

Fig. 7. SCAS of TDC simulation results with model uncertainties.

\subsection{Landing simulation}

The TDC landing simulation was conducted with the full nonlinear aircraft simulation model including the nonlinear aerodynamic coefficients. Three kinds of simulation cases are considered as following;

Case 1: without disturbances (no wind shear, gust, and turbulence)

Case 2: with moderate disturbances (with turbulence, no wind shear, and gust)

Case 3: with strong disturbances (combination of gust, turbulence, and wind shear)

For Case 1, a landing simulation without disturbances is presented. In this case, the variation of the aerodynamic coefficients due to the flap deflection is considered as uncertainties. The landing scenario considered here is as follows:

1) Level flight at $100 \mathrm{knot}$, angle of attack $8.62^{\circ}$

2) Glide slope capture at altitude of $250 \mathrm{ft}$

3) Flap deflection at altitude of $150 \mathrm{ft}$ 

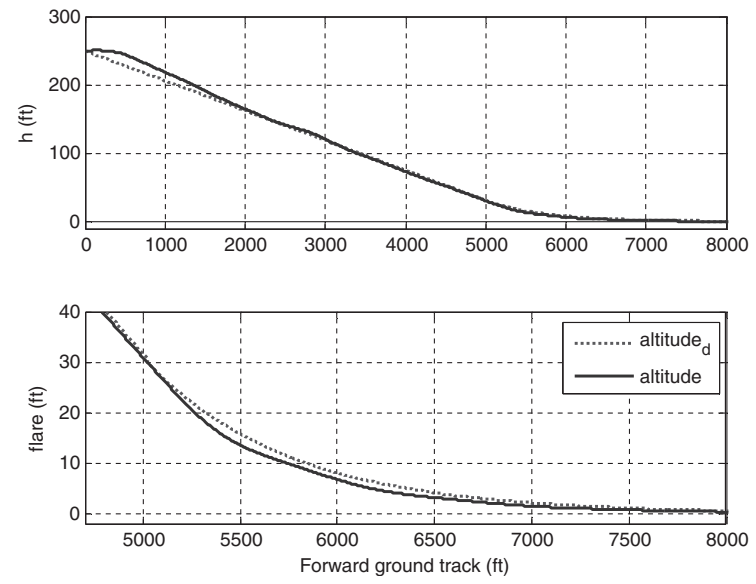

Fig. 8. Longitudinal landing flight path (Case 1).
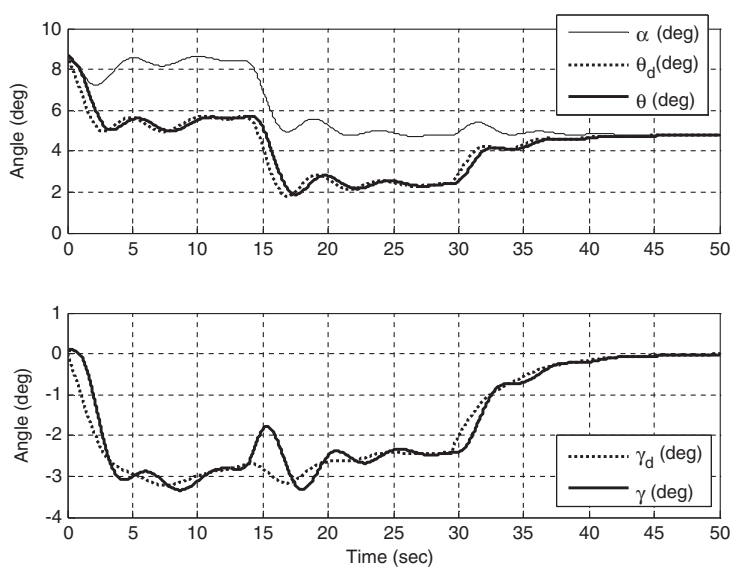

Fig. 9. Aircraft pitch and angle of attack (Case 1).
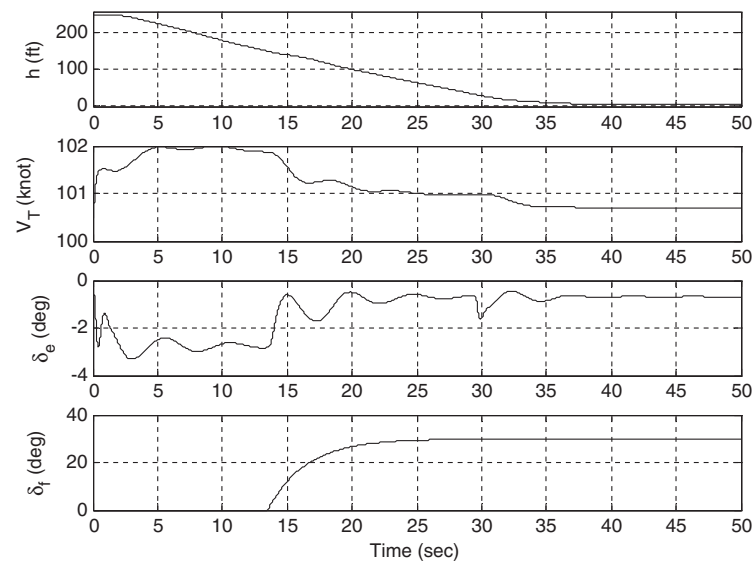

Fig. 10. Control inputs and airspeed (Case 1).

4) Flare at altitude of $30 \mathrm{ft}$

5) Touch-down

Figure 8 shows the tracking performance of the glide slope and flare altitude trajectory corresponding to the ground track. Figure 9 shows the angle of attack with the desired values and response results for pitch and flight path angle. The resultant time histories of altitude, airspeed, and control inputs (elevator and flap angle) are shown in Fig. 10.
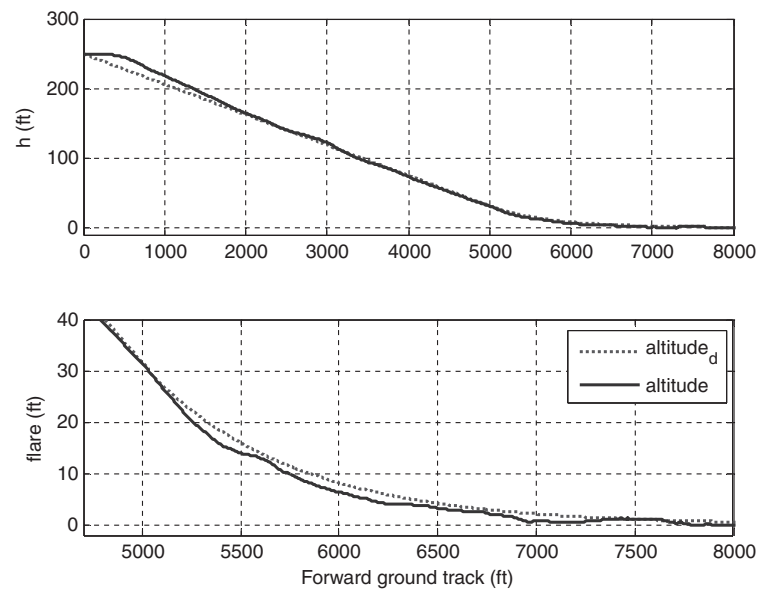

Fig. 11. Longitudinal landing flight path (Case 2).
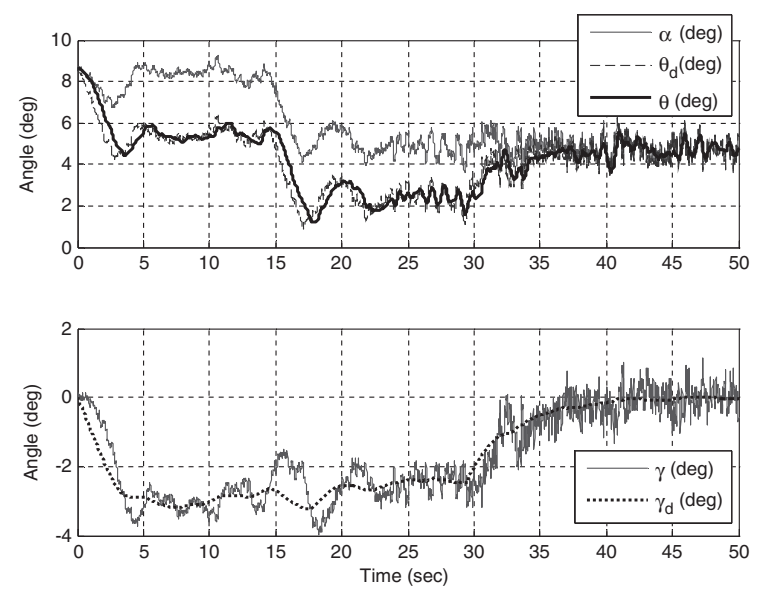

Fig. 12. Aircraft pitch and angle of attack (Case 2).
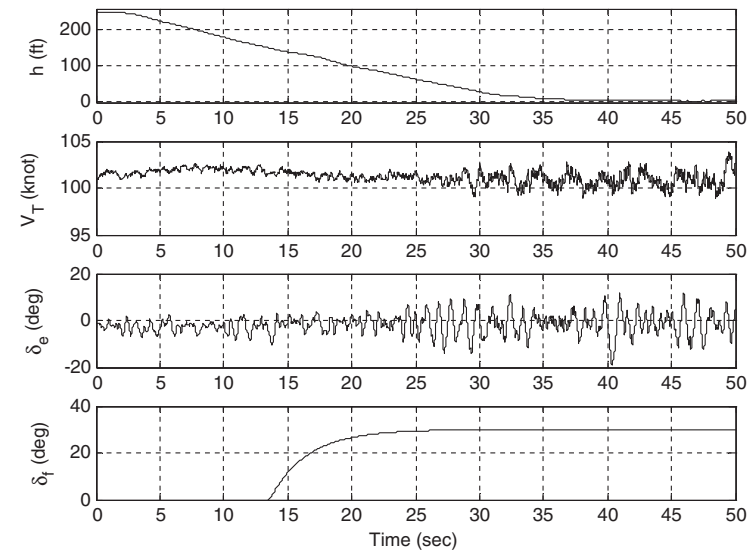

Fig. 13. Control inputs and airspeed (Case 2).

In this case, the landing altitude trajectory tracking is smooth and clean. The elevator control input provides reasonable movement. In Fig. 8, the deviation of flight path angle $\gamma$ is caused by the disturbed angle of attack $\alpha$ due to flap deflection at $15 \mathrm{~s}$. The simulation result shows that TDC auto landing scheme works successfully, tracking desired altitude, considering the angle of attack as disturbance.

Case 2 only considers moderate turbulence and robustness of the controller is evaluated for this disturbance. The inten- 


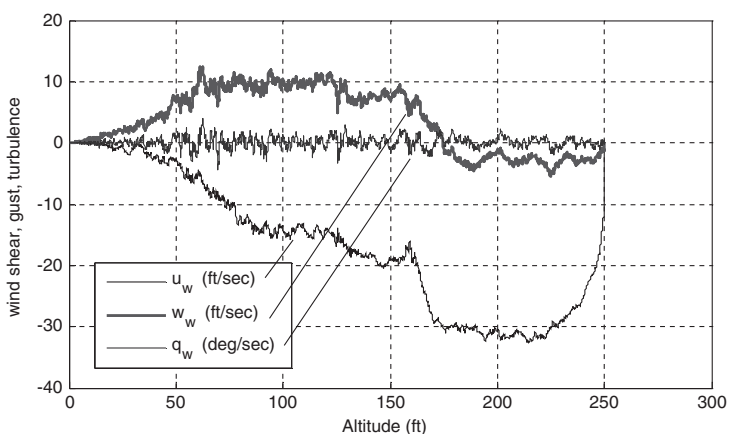

Fig. 14. Wind shear, gust, turbulence profile (Case 3).
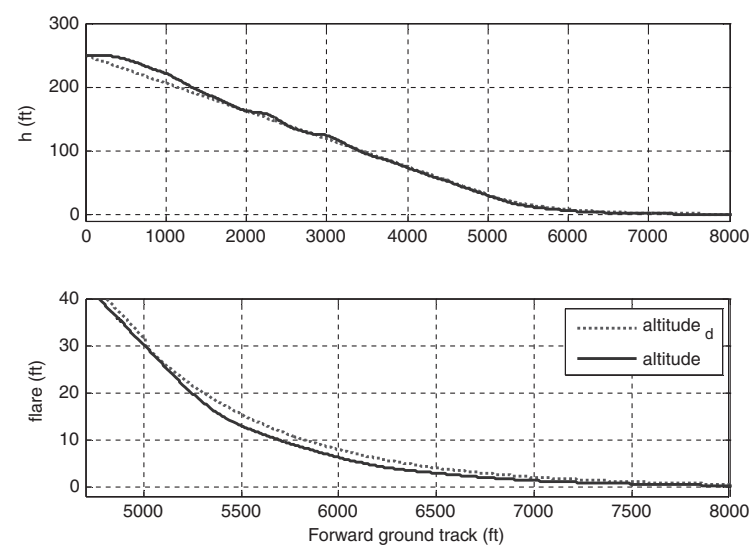

Fig. 15. Longitudinal landing flight path (Case 3).

sity of the turbulence is $3.0-3.5 \mathrm{ft} / \mathrm{s}$ for the horizontal $\left(u_{\mathrm{w}}\right)$ and vertical velocity $\left(w_{\mathrm{w}}\right)$ and about $5.0 \mathrm{deg} / \mathrm{s}$ for the pitch rate $\left(q_{\mathrm{w}}\right)$ during the entire landing phase. Figures 11-13 show the landing trajectories, state, and control variables with respect to time. In the flare phase, the altitude trajectory is reasonable with respect to moderate turbulence.

Finally, Case 3 shows the strong disturbances condition. The wind shear, gust, and turbulence models are combined and then inserted as the strong disturbances during landing simulation. Combined wind profiles along the altitude are shown in Fig. 14. Compared to the moderate disturbance (Case 2), the intensity of turbulence is stronger and onground wind velocity is zero. ${ }^{11,12)}$

Figures 15-17 show the simulation results for the landing simulation at strong disturbances. As confirmed by each result, the proposed auto-landing controller follows the given trajectories successfully and shows good performance and robustness to external disturbances.

\section{Conclusions}

A TDC scheme is proposed for the longitudinal autolanding guidance assuming airspeed is constant, sideslip angle and yaw rate are zero, and angle of attack is a disturbance. SCAS simulation shows that the TDC is robust to model uncertainties such as variation in $\mathrm{CG}$, moment of inertia, and aerodynamic coefficient uncertainties due to flap deflection and one stuck elevator control surface. Furthermore, landing simulation confirms that the proposed TDC
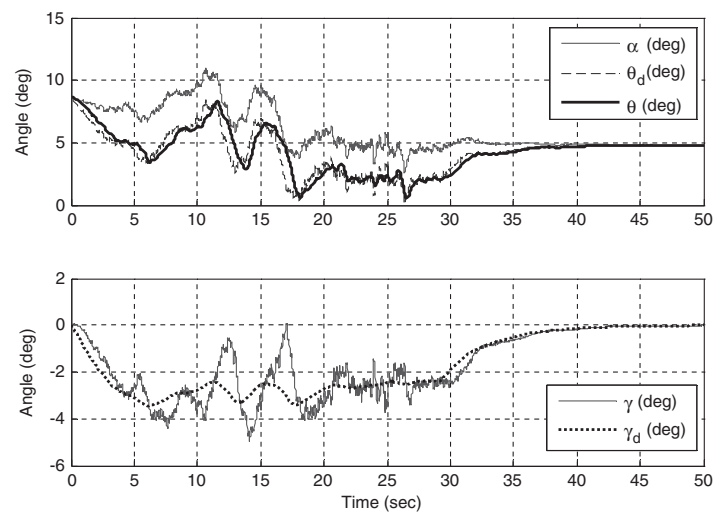

Fig. 16. Aircraft pitch and angle of attack (Case 3).
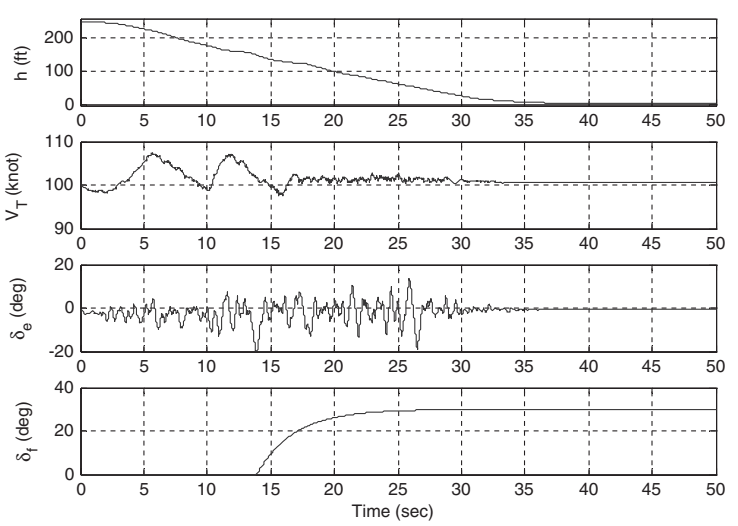

Fig. 17. Control inputs and airspeed (Case 3).

is robust to disturbances, such as angle of attack variation, gust, wind shear, and turbulence.

Specifically, the proposed TDC auto-landing guidance law does not require an accurate system model and properties. The required model properties are only estimated moment of inertia $\hat{I}_{y}$ and estimated control moment $\hat{M}_{\delta_{\mathrm{e}}}$, so the control law is very easy to implement. In addition, the TDC guidance law does not require external information such as ILS (Instrument Landing System) signals because it uses altitude and position information. Therefore, the TDC has potential applications in auto-landing and navigation guidance control for UAV.

\section{References}

1) Youcef-Toumi, K. and Ito, O.: A Time Delay Controller Design for Systems with Unknown Dynamics, ASME J. Dyn. Sys. Meas. Control, 112 (1990), pp. 133-142.

2) Chin, S. M., Lee, C. O. and Chang, P. H.: An Experimental Study on the Position Control of An Electrohydraulic Servo System Using Time Delay Control, Control Engineering Practice, 2 (1994), pp. 41-48.

3) Jin, M., Kang, S. H. and Chang, P. H.: Robust Compliant Motion Control of Robot with Nonlinear Friction Using Time-Delay Estimation, IEEE Trans. Industrial Electronics, 55 (2008), pp. 258-269.

4) Ju, H. S. and Tsai, C. C.: Longitudinal Auto-landing Controller Design via Adaptive Backstepping, Int. J. Adapt. Control Signal Process, 22 (2008), pp. 640-666.

5) Ha, C., Choi, H. S. and Kim, B. S.: Design of Glide Slope Capture Logic Using Model Inversion, International Conference on Control, Automatics and System, 2001. 
6) Stevens, B. L. and Lewis, F. L.: Aircraft Control and Simulation, John Willey \& Sons, New York, 1992, pp. 80-82.

7) Blakelock, J. H.: Automatic Control of Aircraft and Missiles, 2nd ed., John Willey \& Sons, New York, 1991, pp. 88-97.

8) Chang, P. H. and Lee, J. W.: A Model Reference Observer for TimeDelay Control and Its Application to Robot Trajectory Control, IEEE Trans. Control Sys. Technol., 4 (1996), pp. 2-10.

9) Chang, P. H. and Park, S. H.: On Improving Time-delay Control under Certain Hard Nonlinearities, Mechatronics, 13 (2003), pp. 393-412.
10) Chang, P. H., Park, B. S. and Park, K. C.: An Experimental Study on Improving Hybrid Position Force Control of a Robot Using Time Delay Control, Mechatronics, 6 (1996), pp. 915-931.

11) Liao, F., Wang, J. L., Poh, E. K. and Li, D.: Fault-tolerant Robust Automatic Landing Control Design, J. Guid. Control Dynam., 28 (2005), pp. 854-871.

12) Malaek, S. M. B., Sadati, N., Izadi, H. and Pakmehr, M.: Intelligent Autolanding Controller Design Using Neural Networks and Fuzzy Logic, IEEE 5th Asian Control Conference, 2004. 